\title{
Missed Opportunities to Vaccinate Older Adults in Primary Care
}

\author{
Mary Patricia Nowalk, PhD, RD, Richard Kent Zimmerman, MD, MPH, \\ Stephanie M. Cleary, and Richard D. Brueblman, MD
}

Objective: During the 2000 to 2001 influenza season, distribution of influenza vaccine was delayed, and national self-reported vaccination rates declined. The purposes of this study were to characterize missed opportunities for adult vaccinations and assess the impact of the vaccine delay on missed opportunities for influenza vaccination as recorded in medical records.

Methods: In a cross-sectional analysis, medical record data from 217 adult patients aged $\geq 65$ years in primary care practices that received influenza vaccine supplies late in 2000 were used to assess rates and missed opportunities to vaccinate. Missed opportunities were defined as visits in which there was no record that vaccine had been given, discussed, or refused by the patient.

Results: During the mean study period of $37.1 \pm 5.7$ months, patients averaged $12.1 \pm 5.9$ visits to their primary care physician's office. Medical records indicated that $75 \%$ of patients had received pneumococcal polysaccharide vaccine (PPV) and 30\% had received tetanus toxoid from 1991 to 2001; 81\% had received at least one influenza vaccine in the previous 4 seasons. During the 2000 to 2001 influenza season, influenza vaccination rates declined significantly to $41 \%$ from $57 \%$ in 1999 to 2000 . Overall missed opportunities to vaccinate during the study period averaged $3.4 \pm 3.0$ for influenza vaccine, $10.7 \pm 7.3$ for pneumococcal vaccine, and $10.8 \pm 5.9$ for tetanus toxoid. During the delay season, the number of visits increased, but missed opportunities to vaccinate also increased significantly, even after vaccine supplies had been received.

Conclusions: Missed opportunities to vaccinate occur frequently and vaccine shortages create additional challenges to adult vaccination. Missed opportunities may be minimized and maintenance of accurate adult immunization records may be achieved by assessing and recording vaccination status at each visit, regardless of vaccine availability. By so doing, providers can easily convey the message to their patients that immunizations are an important part of their care. (J Am Board Fam Pract 2005; 18: 20-7.)

Previous research into barriers to adult vaccination indicates that physician recommendation is an important factor related to vaccination status, ${ }^{1-5}$ and most physicians recognize the value of immuniza-

Submitted, revised, 8 November 2004.

From the Department of Family Medicine and Clinical Epidemiology, University of Pittsburgh School of Medicine, Pittsburgh, PA (MPN, RKZ, SMC), Department of Behavioral and Community Health Sciences, University of Pittsburgh Graduate School of Public Health, Pittsburgh, PA (RKZ), and Renaissance Family Practice, Pittsburgh, PA (RDB). Address correspondence to Mary Patricia Nowalk, PhD, RD, Department of Family Medicine and Clinical Epidemiology, University of Pittsburgh School of Medicine, 3518 Fifth Avenue, Pittsburgh, PA 15261 (e-mail: tnowalk@ pitt.edu).

This publication/project was made possible through a Cooperative Agreement between the Centers for Disease Control and Prevention (CDC) and The Association of Teachers of Preventive Medicine (ATPM), award TS-0550$16 / 16$; its contents are the responsibility of the authors and do not necessarily reflect the official views of the CDC or ATPM. tion for disease prevention. ${ }^{6}$ Yet for adults $\geq 65$ years, overall vaccination rates of $63 \%$ for influenza and $55 \%$ for pneumococcal polysaccharide vaccine $(\mathrm{PPV})^{7}$ remain substantially below the $90 \%$ goal set by Healthy People 2010. ${ }^{8}$ Therefore, influenza and pneumonia are the fifth leading cause of death among older adults and contribute significantly to excess morbidity and hospitalization. ${ }^{9}$ Further complicating this situation are potential fluctuations in vaccine supply caused by production and regulatory difficulties, as occurred in the 2000 to 2001 influenza season. ${ }^{10}$ During that season, a highly publicized delay in distribution of influenza vaccine resulted in providers receiving their supplies in diminished amounts, up to 3 months later than usual, and frequently at higher cost than in previous years. Given the seasonal nature of influenza vaccination and the narrow window of opportunity for vaccinating, a 3-month delay might have a major impact on vaccination rates. In fact, nation- 
wide, self-reported influenza vaccination rates for adults $\geq 65$ years declined from $65.7 \%$ in 1999 to $64.3 \%$ in $2000 .^{7}$ However, the impact of the delay on primary care practice, on vaccination rates as reported in primary care medical records, and on opportunities to vaccinate in the primary care office is unknown.

The purposes of this study were to assess adult immunization rates and missed opportunities to be vaccinated as reported in medical records and compare influenza immunization rates during the 2000 to 2001 influenza vaccine delay with rates in nondelay years.

\section{Methods \\ Subjects}

Two-stage, stratified random cluster sampling from billing records of family practices in the Pittsburgh metropolitan area was used to generate a sample for a survey regarding patient attitudes and beliefs about adult immunizations. Results from the patient telephone interview have been published. ${ }^{11,12}$ Seven of those practices were then specifically selected for this study because they received influenza vaccine supply late (December) in the 2000 to 2001 influenza season compared with 1998 to 1999,1999 to 2000 , and 2001 to 2002, in which supplies were received in October. Thus, the participants of this study are a subsample of those who were randomly sampled from the practices and had agreed to a telephone survey and medical record review.

Eligibility criteria for the survey were age $\geq 65$ years as of 1 October 1999 (to ensure eligibility for influenza vaccine in the 1999 to 2000 season), an office visit on or after 1 October 1998, and ability to answer survey questions in either English or Spanish. Exclusion criteria included residence in a nursing home; being deaf or homeless; having severe psychosis, senility, or dementia; or not living in the Pittsburgh metropolitan area.

A personalized introductory letter and a letter from the patient's physician endorsing the project and encouraging participation, as well as a letter from the research team, were sent to each of the sampled patients. An honorarium was offered to encourage participation. The Institutional Review Board of the University of Pittsburgh approved this project.

\section{Medical Record Review}

A customized electronic spreadsheet was created for direct data entry using a laptop computer. A code book was developed to guide the medical record reviewer. The following data were collected: sex, age, date of first visit to practice, and, for each visit, date; type; name of clinician seen; and immunizations given, discussed, or refused as reported in the progress notes or on a health maintenance flow sheet (HMFS). None of the practices had electronic medical records. Medical record reviews took place between December 2001 and April 2002. Progress notes and health maintenance flow sheets for individual visits dated 1 October 1998 through 31 December 2001 were examined for administration of vaccines so that 4 influenza vaccination seasons were included. In addition, documentation of receipt of PPV and tetanus toxoid back through 1991 was examined, using progress notes and the HMFS, if available. However, individual visit data before 1 October 98 were not recorded. The resulting data file contained approximately 4000 lines of individual visit data.

\section{Data Preparation}

The raw data contained one or more lines of visit data for each participant (1 line/visit), which were reduced to a single data line for each participant. A research assistant was responsible for collating the data for each patient, creating a summary database with total visits, total acute (eg, viral infection), chronic (eg, hypertension follow-up), and preventive (eg, annual physical) visits, number of visits with the study-assigned primary care provider (PCP), demographic variables and immunizations given, discussed and refused as recorded on progress notes and HMFS. Vaccine administration recorded in either the progress notes or the HMFS was accepted. Ninety-four percent of patient charts had HMFS. Cancelled appointments or laboratoryonly visits were not included. Patients who were not seen during the 39-month window were not included.

\section{Determination of Missed Opportunities}

For PPV and tetanus, missed opportunities for the 39 month study period for patients without tetanus and pneumococcal vaccinations were defined as total visits minus any visits in which vaccine was discussed with or refused by the patient. To be conservative, patients vaccinated at any time during 
the study period were excluded from the missed opportunity analyses. Although this method underestimates missed opportunities, it was chosen because pneumococcal or tetanus vaccines given between 1991 and October 1998 could not be attributed to specific visit dates.

Because influenza vaccine is given annually and $\leq 4$ seasons were assessed, missed opportunities were calculated separately for each part [ie, early (October and November) and late (December through February)] of each season. Missed opportunities for the 39-month study period were defined as visits during each part of the influenza season in which influenza vaccine was neither given, discussed by the physician, nor refused by the patient. Visits that took place other than during the influenza vaccination season were not included in these missed opportunities calculations.

\section{Statistical Analyses}

The data were transferred into an SPSS (SPSS Inc., Chicago, IL) database for analysis. Analysis of variance, $\chi^{2}$ and $t$ tests were used to determine significant differences between groups, and repeated measures analysis of variance was used to determine differences across influenza seasons. $P$ was set at $\leq$ .05 for all tests of significance.

\section{Results}

In the original sampling scheme, 300 patients in practices with late receipt of influenza vaccine in 2000 to 2001 were selected for contact, and 277 (92\%) were reached and consented to be interviewed. Of those, $222(80 \%)$ consented to medical record review and 217 (78\%) had usable data available. Demographic characteristics of the sample are shown in Table 1. Participants, at an average age of 75 years, were well within the recommended ages for all 3 vaccines. Bivariate analyses with age categories and gender as separate grouping variables resulted in no meaningful differences between groups. Therefore, no further analyses based on age or gender were conducted.

The number of patients whose medical records indicated that they had received an annual influenza vaccine or had ever received pneumococcal and tetanus vaccines were significantly below national goals of $90 \%$. Although patients had an average $3.9 \pm 2.0$ total visits per year, only $10 \%$ of the sample was considered to be fully immunized (ie,
Table 1. Demographic Characteristics, Visits and Vaccination Status

\begin{tabular}{lc}
\hline Characteristic & $\mathrm{n}=217$ \\
\hline Age (years) (mean \pm SD) & $74.9 \pm 5.3$ \\
Sex (\% male) & 43.8 \\
Number of months in study (mean \pm SD) & $37.1 \pm 5.7$ \\
Number of influenza seasons available & $3.8 \pm 0.5$ \\
$\quad$ (mean \pm SD) & $12.1 \pm 5.9$ \\
Total visits (mean \pm SD) & $8.6 \pm 5.6$ \\
Primary care physician visits (mean \pm SD) & $70.6 \pm 28.5$ \\
$\%$ Primary care physician visits (mean \pm SD) & $1.1 \pm 1.5$ \\
Acute visits (mean \pm SD) & $8.9 \pm 5.6$ \\
Chronic visits (mean \pm SD) & $2.1 \pm 1.8$ \\
Preventive visits (mean \pm SD) & $81.1^{*}$ \\
Ever received influenza vaccine (\%) & 23.5 \\
Always received influenza vaccine (\%) & 75.1 \\
Ever received pneumococcal vaccine (\%) & 29.5 \\
Ever received tetanus toxoid (\%) & 10.1 \\
Fully immunized ${ }^{\dagger}$ (\%) & 7.4 \\
Received no vaccines (\%) &
\end{tabular}

There were no significant differences by age group or sex.

* Percentage receiving influenza vaccine in 2000 to 2001 season was significantly lower than all three other seasons, by $\mathrm{McNe}-$ $\operatorname{mar} \chi^{2}$ test $(P<.001)$.

${ }^{\dagger}$ Received one influenza vaccine per season available and the pneumococcal vaccine and tetanus toxoid within the appropriate interval.

having ever received both pneumococcal and tetanus vaccines and received annual influenza vaccine) and $7 \%$ had not received any vaccines during the period of medical record review.

Table 2 indicates the number of visits of different types and components of missed opportunities associated with vaccination status. Receiving influenza vaccine at least once in 4 years was associated with significantly higher numbers of total visits and visits with the primary care physician (as opposed to another provider in the practice). Correlations between number of visits during influenza season and receiving influenza vaccine were significant overall $(r=0.45 ; P<.01)$ and varied slightly from year to year: 1998 to $1999 r=0.34 ; 1999$ to $2000 \mathrm{r}$ $=0.33 ; 2000$ to $2001 \mathrm{r}=0.31$; and 2001 to 2002 $r=0.35$.

Given that vaccination rates were quite low, we wished to examine whether this was because physicians did not address vaccination with patients, did not record discussing vaccination, or because patients refused vaccination. Table 2 also shows the average number of visits at which physicians discussed each vaccine, refusals by patients, and 


\begin{tabular}{|c|c|c|c|c|c|c|c|}
\hline & \multirow{2}{*}{$\begin{array}{l}\text { Never Received an } \\
\text { Influenza Vaccine } \\
(1998 \text { to 2001) } \\
(\mathrm{n}=41)\end{array}$} & \multirow{2}{*}{$\begin{array}{l}\text { Received } 1 \text { to } 3 \\
\text { Influenza Vaccines } \\
(1998 \text { to } 2001) \\
(\mathrm{n}=125)\end{array}$} & \multirow{2}{*}{$\begin{array}{l}\text { Received } 1 \text { Influenza } \\
\text { Vaccine/Season } \\
(1998 \text { to } 2001) \\
(\mathrm{n}=51)\end{array}$} & \multicolumn{2}{|c|}{$\begin{array}{c}\text { Received } \\
\text { Pneumococcal } \\
\text { Age } \geq 65 \text { years } \\
(1991 \text { to 2001) }\end{array}$} & \multicolumn{2}{|c|}{$\begin{array}{l}\text { Ever Received } \\
\text { Tetanus } \\
\text { (1991 to 2001) }\end{array}$} \\
\hline & & & & $\begin{array}{c}\text { Yes } \\
(\mathrm{n}=163)\end{array}$ & $\begin{array}{c}\text { No } \\
(\mathrm{n}=54)\end{array}$ & $\begin{array}{c}\text { Yes } \\
(\mathrm{n}=64)\end{array}$ & $\begin{array}{c}\text { No } \\
(\mathrm{n}=153)\end{array}$ \\
\hline Total visits & $10.2 \pm 6.0$ & $11.7 \pm 5.9^{\dagger}$ & $14.7 \pm 5.3$ & $12.2 \pm 5.7$ & $12.0 \pm 6.7$ & $13.3 \pm 5.9$ & $11.6 \pm 5.9$ \\
\hline PCP visits & $8.1 \pm 5.9$ & $8.0 \pm 5.2^{\ddagger}$ & $10.5 \pm 5.7$ & $8.6 \pm 5.3$ & $8.7 \pm 6.3$ & $9.4 \pm 5.0$ & $8.3 \pm 5.8$ \\
\hline$\%$ PCP visits & $79.1 \pm 25.8$ & $68.6 \pm 29.0$ & $68.3 \pm 28.7$ & $70.5 \pm 28.6$ & $70.9 \pm 28.4$ & $72.6 \pm 23.9$ & $69.8 \pm 30.3$ \\
\hline MD discussed & $0.15 \pm 0.36$ & $0.14 \pm 0.39$ & & & $0.91 \pm 1.58$ & & $0.67 \pm 1.38$ \\
\hline Patient refused & $0.32 \pm 0.57$ & $0.0 .2 \pm 0.15^{\S}$ & & & $0.31 \pm 0.84$ & & $0.09 \pm 0.44$ \\
\hline $\begin{array}{l}\text { Missed } \\
\text { opportunities }\end{array}$ & $3.4 \pm 3.0$ & $2.18 \pm 2.13^{\mathbb{I}}$ & & & $10.7 \pm 7.3$ & & $10.8 \pm 5.9$ \\
\hline
\end{tabular}

* PCP, primary care physician visits; \% PCP, percentage of visits with primary care physician. All post hoc comparisons used Bonferroni corrections. Total visits for influenza vaccination are those during influenza season only; for pneumococcal and tetanus total visits are year round.

${ }^{\dagger} P<.01$ difference between never received and received 1 to 3 vaccines and between never received and received $1 /$ season.

${ }^{\ddagger} P<.05$ difference between received 1 to 3 vaccines and received $1 /$ season.

$\$ P<.001$ difference between never received and received 1 to 3 vaccines.

${ }^{\mathbb{I}} P<.01$ difference between never received and received 1 to 3 vaccines.

missed opportunities. Patient refusals were more frequent among those who never received influenza vaccine; however, missed opportunities to vaccinate were also significantly higher in this group. Missed opportunities for influenza vaccination were lower than for pneumococcal vaccine and tetanus toxoid. (It should be noted that influenza vaccine is only given during part of the year, whereas pneumococcal vaccine and tetanus toxoid can be given year round.) It is clear that recorded patient refusals do not explain low vaccination rates.

The influenza vaccine shortage and delayed distribution in the 2000 to 2001 season created an opportunity to study the impact of one factor (ie, vaccine availability) on vaccination status. For each influenza vaccination season, the number of visits are shown in Table 3. Those who received influenza vaccine in any of the 4 seasons had signifi- cantly more office visits during the influenza season than those who were not vaccinated. Visits during the peak vaccination period (October and November) of nondelay seasons were significantly higher among the vaccinated than the unvaccinated. In the delay season (2000 to 2001), vaccinated patients visited the primary care office significantly more often later in the season (December-February). Although the total number of visits and discussions about influenza vaccination increased during the 2000 to 2001 influenza season, missed opportunities to vaccinate increased as well (Table 4). During October and November, when vaccine was unavailable, potential missed opportunities to vaccinate were significantly higher during the delay season than the previous or subsequent season, as might be expected. More importantly, missed opportunities during the latter part of the season, December 2000

Table 3. Comparison of Number of Visits (Mean \pm SD) during 4 Influenza Vaccination Seasons

\begin{tabular}{|c|c|c|c|c|c|c|c|c|}
\hline \multirow[b]{2}{*}{ Visits } & \multicolumn{2}{|c|}{1998 to 1999} & \multicolumn{2}{|c|}{1999 to 2000} & \multicolumn{2}{|c|}{2000 to 2001} & \multicolumn{2}{|c|}{2001 to 2002} \\
\hline & $\begin{array}{l}\text { Vaccinated } \\
(\mathrm{n}=106)\end{array}$ & $\begin{array}{c}\text { Not } \\
\text { Vaccinated } \\
(\mathrm{n}=99)\end{array}$ & $\begin{array}{l}\text { Vaccinated } \\
(\mathrm{n}=122)\end{array}$ & $\begin{array}{c}\text { Not } \\
\text { Vaccinated } \\
(\mathrm{n}=91)\end{array}$ & $\begin{array}{c}\text { Vaccinated } \\
(\mathrm{n}=89)\end{array}$ & $\begin{array}{c}\text { Not } \\
\text { Vaccinated } \\
(\mathrm{n}=128)\end{array}$ & $\begin{array}{l}\text { Vaccinated } \\
(\mathrm{n}=119)\end{array}$ & $\begin{array}{c}\text { Not } \\
\text { Vaccinated } \\
(\mathrm{n}=98\end{array}$ \\
\hline Oct-Nov & $1.13 \pm 0.77^{*}$ & $0.35 \pm 0.69$ & $0.99 \pm 0.66^{*}$ & $0.44 \pm 0.69$ & $0.78 \pm 0.96$ & $0.70 \pm 0.77$ & $1.20 \pm 0.91^{*}$ & $0.62 \pm 0.75$ \\
\hline Dec-Feb & $0.89 \pm 0.91$ & $0.78 \pm 0.91$ & $0.93 \pm 0.99$ & $0.68 \pm 0.92$ & $1.06 \pm 1.24^{*}$ & $0.79 \pm 0.88$ & $-^{\dagger}$ & - \\
\hline Oct-Feb & $2.02 \pm 1.23^{*}$ & $1.13 \pm 1.27$ & $1.92 \pm 1.16^{*}$ & $1.12 \pm 1.16$ & $2.37 \pm 1.63^{*}$ & $1.48 \pm 1.17$ & - & - \\
\hline
\end{tabular}

* Difference between vaccinated and unvaccinated is significant at the $P<.001$ level.

${ }^{\dagger}$ Visits in January and February 2002 were not collected. 


\begin{tabular}{|c|c|c|c|c|}
\hline $\begin{array}{l}\text { Discussions, Refusals and Missed } \\
\text { Opportunities to Vaccinate }\end{array}$ & 1998 to 1999 & 1999 to 2000 & 2000 to 2001 & 2001 to 2002 \\
\hline Vaccinated patients, n (\%) & $106(51.7)$ & $122(57.3)$ & $89(41.0)^{*}$ & $119(54.8)$ \\
\hline $\begin{array}{l}\text { Unvaccinated patients with } \geq 1 \text { missed opportunity } \\
\text { during influenza season, } \mathrm{n}(\%)\end{array}$ & $59(59.6)$ & $52(57.1)$ & $90(70.3)$ & $48(49.0)$ \\
\hline Visits Oct-Feb $^{\dagger}$ & $1.5 \pm 1.3$ & $1.6 \pm 1.2$ & $1.8 \pm 1.4^{\S}$ & \\
\hline MD discussed vaccination $^{\dagger}$ & $0.01 \pm 0.10$ & $0.02 \pm 0.13$ & $0.06 \pm 0.27^{\ddagger}$ & $0.02 \pm 0.13$ \\
\hline Patient refused vaccination ${ }^{\dagger}$ & $0.01 \pm 0.07$ & $0.02 \pm 0.14$ & $0.04 \pm 0.22$ & $0.01 \pm 0.01$ \\
\hline Missed opportunities to vaccinate Oct-Nov ${ }^{\dagger}$ & $0.16 \pm 0.50$ & $0.17 \pm 0.46$ & $0.37 \pm 0.66^{\mathrm{TI}}$ & $0.20 \pm 0.46$ \\
\hline Missed opportunities to vaccinate $\mathrm{Dec}-\mathrm{Feb}^{\dagger}$ & $0.37 \pm 0.73$ & $0.27 \pm 0.68$ & $0.41 \pm 0.78^{* *}$ & \\
\hline Missed opportunities to vaccinate $\mathrm{Oct}-\mathrm{Feb}^{\dagger}$ & $0.53 \pm 1.04$ & $0.45 \pm 0.91$ & $0.78 \pm 1.14^{\dagger \dagger}$ & \\
\hline
\end{tabular}

* 2000 to 2001 influenza season was significantly lower than the 1998 to 1999,1999 to 2000 , and 2001 to 2002 seasons $(P<.001)$.

${ }^{\dagger}$ Mean \pm SD.

$\$ 2000$ to 2001 influenza season was significantly higher than the 1999 to 2000 and 2001 to 2002 seasons $(P<.005)$; data were not collected for visits in January and February 2002, precluding comparison analysis.

$¥ 2000$ to 2001 influenza season was significantly higher than the 1999 to 2000 and 2001 to 2002 seasons $(P<.05)$.

II 2000 to 2001 influenza season was significantly higher than the 1999 to 2000 and 2001 to 2002 seasons $(P<.002)$. To show the impact of the shortage year, visits were treated as potential missed opportunities if unvaccinated; although vaccine was not available in these practices, it was available elsewhere in 2000 to 2001 and in other years in these practices.

** 2000 to 2001 influenza season was significantly higher than the 1999 to 2000 season $(P<.05)$; data were not collected for visits in January and February 2002, precluding comparison analysis.

+† 2000 to 2001 influenza season was significantly higher than the 1999 to 2000 season $(P<.001)$; data were not collected for visits in January and February 2002, precluding comparison analysis.

through February 2001 (after vaccine supplies had been received), were significantly higher than the previous season.

\section{Discussion}

Among adults aged $\geq 65$ years, which is the age group we studied, national recommendations are to receive an influenza vaccine once each year, one pneumococcal polysaccharide vaccine, and tetanus toxoid every 10 years. ${ }^{13}$ Specific goals for immunization rates among this age-group are $90 \%$ for influenza and pneumococcal vaccines, as stated in Healthy People $2010^{8}$ and $62 \%$ for tetanus toxoid, as stated in Healthy People $2000^{14}$ (a new goal for tetanus was not subsequently established). In contrast, national self-reported vaccination rates are $63 \%$ for influenza vaccine, $55 \%$ for pneumococcal vaccine, ${ }^{7}$ and $65 \%$ for tetanus toxoid ${ }^{15}$; we found in medical record review rates of $57 \%$ for influenza (highest of the 4 seasons), $75 \%$ for pneumococcal vaccine, and $30 \%$ for tetanus toxoid. These discrepancies warrant further examination of visit patterns and missed opportunities.

We observed significant differences in the number of total and PCP visits between those who were vaccinated against influenza and those who were not and significant positive correlations between number of visits during influenza season and influenza vaccination status. More frequent visits were not significantly related to pneumococcal and tetanus vaccination status. This finding differs from data from the Behavioral Risk factor Surveillance Survey (BRFSS) and the National Health Interview Survey (NHIS), which indicated that vaccination rates for influenza, pneumococcus, and tetanus vaccines all increased with an increasing number of office visits. ${ }^{15}$ This incongruity may be the result of differing methods, in that the rates reported in the BRFSS and the NHIS are both self-reported, contrasting with medical record review in the present study. There are several possible explanations for the differences we observed in the relationships between visit frequency and influenza vaccination and between visit frequency and PPV and tetanus toxoid vaccination.

Patients who visit their physicians more frequently are presumably more compliant with recommendations for scheduling or are sicker and require more frequent visits. Given the finding that vaccinated patients had more frequent visits during the influenza season, it is possible that during influenza season, there is a greater sensitivity to the potential consequences of an influenza infection for these patients. Therefore, influenza vaccine may be 
offered or requested more often. On the other hand, patients might make an appointment for influenza vaccination. The situation with pneumococcal and tetanus vaccines is different in that they can be given at any time and there is no pneumococcal disease or tetanus "season."

The high rates of pneumococcal vaccination compared with rates for tetanus vaccination may be because (1) Medicare reimburses for the cost of administering pneumococcal vaccine but not for preventive administration of tetanus vaccine; ${ }^{16}$ and (2) pneumococcal disease is common among older patients, but tetanus is relatively rare. ${ }^{17}$ Given the many pressures on primary care physicians' time, tetanus vaccination is less likely to be viewed as a priority prevention measure.

Overall, missed opportunities to vaccinate were high for all 3 vaccines, and patient refusals were low, indicating that vaccination status was not being regularly addressed as is recommended in the Standards for Adult Immunization ${ }^{18}$ or that immunization assessment or discussions were not being documented in the medical record.

Further complicating the primary care physician's ability to reduce missed opportunities to vaccinate are shortages of vaccines. In addition to the widespread shortage of influenza vaccine in 2000 to 2001, there was a delay (albeit considerably less severe) in distribution of influenza vaccine in 2001 to 2002 and a shortage of tetanus vaccine from the fourth quarter of 2000 to mid 2002. We observed a clear and significant impact of the influenza vaccine delay of 2000 to 2001 on the vaccination procedures of primary care physicians' offices that received vaccine late. Overall vaccination rates were lower and missed opportunities to vaccinate were significantly higher during the shortage season, both before vaccine supplies had arrived and afterward. Although both the number of visits and the number of discussions of influenza vaccine increased in 2000 to 2001, missed opportunities increased, suggesting that physicians were either not discussing influenza vaccine at every visit or not documenting such discussions.

The Advisory Committee on Immunization Practices recommends that during the influenza season, unvaccinated high-risk adults should be offered vaccination at any contact with regular providers offering ongoing care, ${ }^{19}$ unless there is a clear contraindication, such as a prior adverse reaction or allergy to a vaccine component. Further- more, the Standards for Adult Immunization Practices include routine review of patients' vaccination status by health care professionals. ${ }^{18}$ When vaccine is not available, it makes no sense to offer vaccine. However, a patient can and should be queried as to vaccination status and his or her plans to receive the vaccine at a later time at the physician's office or elsewhere. We have previously reported that patients were more worried about receiving the influenza vaccine in enough time to prevent influenza. ${ }^{11}$ We have also reported that physician recommendation to be vaccinated is a significant predictor of subsequent vaccination. ${ }^{2,3}$ Discussing the vaccine even when it is not available or indicating places where vaccine is available might help to allay fears about timely receipt of vaccine and encourage vaccination when and where vaccine is available. Furthermore, it provides an opportunity for the provider to discuss other prevention techniques (eg, frequent hand washing), containment techniques (eg, stay home if infected), and treatment strategies if the patient should contract influenza (eg, antiviral medication).

It is possible that physicians did in fact discuss vaccination with patients but did not record doing so in the medical record or did not discuss vaccination early in the season when vaccine was not available. Later, however, when vaccine supplies had been received, physicians would have been expected to check vaccination status and record it in the medical record. We have reported previously that in the 2000 to 2001 season, patients reported receiving influenza vaccine less frequently at their regular doctor's office and more frequently at other locations than during the previous influenza season. ${ }^{11}$ Adherence to another of the Standards for Adult Immunization Practices (ie, that "Vaccination records for patients are accurate and easily accessible" ${ }^{18}$ ) would imply that all vaccinations, including those given elsewhere, should be recorded in the patient's medical record.

Increased use of electronic medical records (EMR) could be very helpful in reducing missed opportunities to vaccinate in primary care offices. We have shown that use of the HMFS increases reported vaccination rates. ${ }^{20}$ This may be because the HMFS serves as a prompt to providers or facilitates documentation of vaccination. EMRs could potentially enhance the effect of the HMFS by providing electronic prompts to vaccinate and/or document vaccination, discussion or patient 
refusal. Any member of the health care team could be responsible for checking vaccination status, administering vaccine, and documentation. Standing orders to vaccinate without an individual physician order, a proven technique for improving vaccination rates, ${ }^{21}$ would further streamline vaccination efforts and save the physician time.

\section{Strengths and limitations}

The strengths of this study are that nearly 4000 visits were examined to identify immunization practices among 25 providers and 217 patients in inner city and suburban primary care practices. Because these practices treat patients in a wide range of socioeconomic status groups and include minority patients, we believe that these findings can be generalized to other family practices in nonrural areas. In addition, we recorded immunizations over 4 influenza seasons, including one in which there was a significant delay in distribution of vaccine. Limitations of the data are that there may have been more missed opportunities to vaccinate that occurred at visits before a patient was vaccinated and that were not counted, given that missed opportunities for vaccinated patients were set to zero. Second, data were not collected for January and February 2002, during which time some patients may have been vaccinated or had missed opportunities to be vaccinated. However, even without that information, the year 2000 influenza vaccine delay had a significant impact on immunization practices in primary care offices. Third, by necessity, vaccine refusals relied on documentation in the medical record.

\section{Conclusions}

Vaccine shortages disrupt even the most efficient systems to vaccinate. Physicians may forego assessing vaccination status either because they do not have vaccines available to offer to eligible patients, do not have time to explain the situation, or are unable to state when vaccine will be available. Yet in the long run, the success of primary care office vaccination systems may be improved by continuing to assess and record vaccination status at each visit. This practice will improve primary care by increasing the accuracy of adult immunization records, giving patients the message that immunization is important, and serving as a reminder to vaccinate when vaccine supplies are once again available.

We acknowledge Daniel Chrzanowski, MD, for medical record reviews and Daniel B. Fishbein, MD, of the Centers for Disease Control and Prevention, for his thoughtful comments on the manuscript.

\section{References}

1. Zimmerman RK Nowalk MP, Bardella IJ, et al. Physician and practice factors related to influenza vaccination among the elderly. Am J Prev Med 2004;26: $1-10$.

2. Zimmerman RK, Santibanez TA, Janosky JE, et al. What affects older patients' influenza vaccination behavior? An analysis from inner-city, suburban, rural, and Veterans Affairs practices. Am J Med 2003; 114:31-8.

3. Zimmerman RK, Santibanez TA, Fine MJ, et al. Barriers and facilitators of pneumococcal vaccination among the elderly. Vaccine 2003;21:1510-7.

4. Nichol KL, Lofgren RP, Gapinski J. Influenza vaccination. Knowledge, attitudes, and behavior among high-risk outpatients. Arch Intern Med 1992;152: $106-10$.

5. Nichol KL, MacDonald R, Hauge M. Factors associated with influenza and pneumococcal vaccination behavior among high-risk adults. J Gen Intern Med 1996;11:673-7.

6. Nichol KL, Zimmerman RK. Generalist and subspecialist physicians' knowledge, attitudes and practices regarding influenza and pneumococcal vaccinations for elderly and other high-risk patients: a nationwide survey. Arch Intern Med 2001;161:2702-8.

7. National Center for Health Statistics. Early release of selected estimates based on data from the JanuarySeptember National Health Interview Survey (NHIS) [monograph on the Internet]. Atlanta (GA): Centers for Disease Control and Prevention; 2003 [cited 2003 May 1]. Available from: http://www.cdc. gov/ nchs/about/major/nhis/released200303.htm\#5.

8. Healthy People 2010. Understanding and improving health and objectives for improving health, 2nd ed. Washington DC: US Department of Health and Human Services; 2000. Available from: http://www. healthypeople.gov/Document/tableofcontents.htm

9. Minino AM, Arias E, Kochanek KD, Murphy SL, Smith BL. Deaths: final data for 2000. Natl Vital Stat Rep 2002;50:1-119.

10. Fukuda K, O'Mara D, Singleton J. How the delayed distribution of influenza vaccine created shortages in 2000 and 2001. Pharmacy Ther 2002;27:235-42.

11. Santibanez TA, Nowalk MP, Zimmerman RK, Bruehlman R. Effect of the year 2000 influenza vaccine delay on elderly patients' attitudes and beliefs. Prev Med 2003;37:417-23.

12. Nowalk MP, Zimmerman RK, Shen S, Jewell IJ, 
Raymund M. Barriers to pneumococcal and influenza vaccination in older community-dwelling adults (2000-2001). J Am Geriatr Soc 2004;52:25-30.

13. Zimmerman RK, Middleton DB, Burns IT, Clover RD. Routine vaccines across the life span. J Fam Pract 2003;52:S1-21.

14. Healthy People 2000: Midcourse review and 1995 revisions. Washington DC: US Department of Health and Human Services; 1995.

15. Singleton JA, Greby SM, Wooten KG, Walker FJ, Strikas R. Influenza, pneumococcal, and tetanus toxoid vaccination of adults-United States, 1993-7. MMWR CDC Surveill Summ 2000;49:39-62.

16. Medicare.gov [homepage on the Internet]. Washington DC: Centers for Medicare and Medicaid Services; 2003 [updated 2004 Nov 18; cited 2003 Nov 24]. Your Medicare coverage; [about 3 screens]. Available from: http://www.medicare.gov/coverage/ home.asp
17. Bardenheier B, Prevots DR, Khetsuriani N, Wharton M. Tetanus surveillance-United States, 19951997. MMWR CDC Surveill Summ 1998;47:1-13.

18. Poland GA, Shefer AM, McCauley M, et al; National Vaccine Advisory Committee, Ad Hoc Working Group for the Development of Standards for Adult Immunization Practices. Standards for adult immunization practices. Am J Prev Med 2003;25: $144-50$.

19. Prevention and control of influenza: recommendations of the Advisory Committee on Immunization Practices (ACIP). MMWR Recomm Rep 1999; 48(RR-4):1-28.

20. Nowalk MP, Zimmerman RK, Feghali J. Missed opportunities for adult immunization in diverse primary care office settings. Vaccine 2004;22:3457-63.

21. Gyorkos TW, Tannenbaum TN, Abrahamowicz M, et al. Evaluation of the effectiveness of immunization delivery methods. Can J Public Health 1994;85 Suppl 1:S14-30 